\title{
Adipogenic differentiation of human adipose- derived mesenchymal stem cells regulated by microRNA-26b-5p/TCF-4
}

\section{Yadong Luo}

the Xuzhou Clinical College of Xuzhou Medical University

Huan Ji

Nanjing Medical University

Yan Cao

Nanjing Medical University

Xu Ding

Nanjing Medical University

Meng Li

Nanjing Medical University

Haiyang Song

Nanjing Medical University

Sheng Li

Nanjing Medical University

Chenxing Wang

Nanjing Medical University

Heming Wu

Nanjing Medical University

Jian Meng

Xuzhou Clinical College of Xuzhou Medical University

Hongming Du ( $\nabla$ dhm_010@sina.com )

Affliated Stomatologiacl Hospital of Nanjing Medical University https://orcid.org/0000-0002-0909-

0773

\section{Research}

Keywords: hADMSCs, adipogenic differentiation, Wnt/ $\beta$-catenin pathway, miRNA-26b-5p, TCF-4

Posted Date: February 13th, 2020

DOl: https://doi.org/10.21203/rs.2.23419/v1 
License: (c) (i) This work is licensed under a Creative Commons Attribution 4.0 International License. Read Full License 


\section{Abstract}

Background: Our study was designed to investigate the role of miR-26b-5p on TCF-4, affecting the adipogenic differentiation of human adipose-derived mesenchymal stem cells (hADMSCs). METHODS: The adipogenic differentiation of hADMSCs was induced by adipogenic medium for 6 days (d). Bioinformatic and dual-luciferase analyses were used to confirm the relationship between TCF-4 and miR26b-5p. Immunofluorescence was used to detect the effect of miR-26b-5p on TCF- 4 and $\beta$-catenin in hADMSCs transfected with miR-26b-5p mimic and inhibitor. Mimic, inhibitor, and small interfering RNA (siRNA) transfected in hADMSCs to against LEF1 and $\beta$-catenin. Quantitative real-time PCR and western blotting were used to examine the adipogenic markers and Wnt/ $\beta$-catenin pathway at the mRNA and protein levels, respectively. Immunofluorescence was performed to locate $\beta$-catenin. RESULTS: hADMSCs could differentiate toward adipocytes by the adipogenic medium. The results of bioinformatic and dualluciferase analyses show that TCF-4 is a potential target of miR-26b-5p. The immunofluorescence intensity of TCF 4 and $\beta$-catenin were inhibited by miR-26b-5p in hADMSCs. Overexpression of miR-26b-5p promotes the adipogenic differentiation of hADMSCs. Overexpression of TCF- 4 and $\beta$-catenin inhibits the adipogenic differentiation of hADMSCs. The adipogenic differentiation of hADMSCs that promoted by knocking down TCF4 could be weakened by low-expression of miR-26b-5p. The stimulative effect of $\beta$ catenin low-expression in adipogenic differentiation was inhibited by miR-26b-5p inhibitor. Conclusions: miR-26b-5p is a negative regulator to inhibit TCF-4 directly, and then inactivated Wnt/ $\beta$-catenin pathway, which promotes the adipogenic differentiation of hADMSCs in vitro.

\section{Introduction}

Trauma and tumors occurred in the oral and maxillofacial region may cause facial soft tissue defects, which is a common problem faced by oral and maxillofacial and plastic surgeons. Flap transplantation, artificial material, and adipose tissue transplantation are often used to reconstruct defects in soft tissue defects of the oral and maxillofacial region [1, 2]. But these methods have some application-level problems. Flap transplantation caused tissue damage and even to defects in the donor site [3]; artificial implant materials usually caused rejection reaction [4]; adipose tissue transplantation faced early absorption, sometimes the patients need a surgical treatment many times[5].

The development of tissue engineering technology provides a new method for repairing soft tissue defects[6], and one of key points is the research of seed cells[7]. Zuk et al. first verified that adipose stem cells have the multi-directional differentiation potential for differentiation into adipocytes, osteoblasts, chondrocytes and myocytes, and become an important seed cell source for tissue regeneration[8]. Adipose-derived mesenchymal stem cells (ADMSCs) have sufficient sources, simple acquisition, high yield, rapid proliferation, multi-directional differentiation potential, can secrete various cytokines, promote angiogenesis, exert immunomodulatory effects, and recruit other cells[9]. Research on ADMSCs as seed cells has become one of the focuses of soft tissue repair and reconstruction. 
How to promote adipogenic differentiation of ADMSCs and avoid fat absorption has become a key area of orthopedic research. Many studies have shown that Wnt/ $\beta$-catenin plays a critical regulatory role in the adipogenic differentiation of adipose-derived mesenchymal stem cells. Wnt3a blocks the differentiation of mouse adipose precursor cell line 3T3-L1 into adipocytes by inhibiting peroxisome proliferatoractivated receptor Y (PPARY) expression [10]. LPR-6-deficient mouse fibroblasts spontaneously differentiate into adipocytes [10]. The above results indicate that $\mathrm{Wnt} / \beta$-catenin plays a negative regulatory role in adipogenic differentiation.

Current research confirms that microRNAs are involved in the regulation of a variety of biological processes, which can affect the adipogenic differentiation process of adipose-derived mesenchymal stem cells by regulating signaling pathways. Some microRNAs involved in adipogenic differentiation, such as hsa-miR-15a-5p, hsa-miR-27a-3p, hsa-miR-106b-5p, miR-17-5p, miR-17, etc., can be promoted adipogenic differentiation of ADMSCs by different signaling pathways[11, 12, 13]. Although many studies have revealed that microRNAs regulate the adipogenic differentiation of adipose stem cells through the Wnt/ $\beta$-catenin signaling pathway, the diversity of microRNAs and the diversity of their target genes. Research needs to go further.

hsa-miR-26b-5p was upregulated in hADMSCs during adipogenic differentiation in our pre-experiment. The bioinformatic analysis indicated that the target of hsa-miR-26b-5p is most likely TCF4 (T cell factor 4 , Gene TCF7L2) in the Wnt/ $\beta$-catenin pathway. We hypothesize that down-regulating has-miR-26b-5p activates the Wnt/ $\beta$-catenin pathway, thereby inhibiting adipogenic differentiation of hADMSCs. This study intends to validate this hypothesis by cell biology and molecular biology methods (Fig. 1.).

\section{Materials And Methods}

\section{The Obtain method and characterization of hADMSCs}

The obtain method and characterization of hADMSCs were illustrated in our previous study [14].

\section{The adipogenic differentiation of hADMSCs}

hADMSCs were seeded into 6-well plates at a density of $9000 / \mathrm{cm}^{2}$.After $48 \mathrm{~h}$, the adipogenic medium was added into each well in 6-well plates, and the medium changed every $2 \mathrm{~d}$. The control groups were just cultured in Alpha-modified Eagle's medium(a-MEM; Gibco BRL, USA) with 10\% fetal bovine serum (FBS; Gibco BRL, USA). Both the adipogenic group and the control group were cultured at $37^{\circ} \mathrm{C}$ and $5 \%$ $\mathrm{CO}_{2}$ incubator for $4 \mathrm{~d}$. On the fourth day, hADMSCs were collected for Microarray for miRNA, immunofluorescence, Real-time Quantitative PCR (qPCR) and western blot analyses.

\section{Bioinformatics Analysis}

We analyze the conservative characters of miR-26b-5p, binding site in $3^{\prime} U T R$ for TCF7L2, and the target of miR-26b-5p. Based on the results of the 3 following databases: TargetScan (http://www.targetscan.org), 
used the following 3 databases to confirm miRWalk (http://mirwalk.umm.uni-heidelberg.de) and miRBase(http://www.mirbase.org).

\section{RNAs and Transfection}

In this study, we constructed some genetic research tools to change the expression of some genes, they were listed below. TCF7L2 expression plasmid (EX-TCF7L2), TCF7L2 siRNA (siTCF7L2), negative control of expression plasmid (EX-Ctrl), negative control of siRNA (siR-Ctrl), miR-26b-5p mimic, miR-26b-5p inhibitor, negative control of miR-26b-5p mimic (miR-Ctrl mimic) and negative control of miR-26b-5p inhibitor (miR-Ctrl inhibitor), TCF7L2 3' UTR-Wild Type (wt-TCF7L2), TCF7L2 3' UTR-Mutant (mu-TCF7L2). They were transfected into the 4th-passage hADMSCs respectively following manufacturer's manual by Lipofectamine $^{\mathrm{TM}} 2000$ Transfection Reagent (Invitrogen, USA). The miR-26b-5p mimic, inhibitor, siTCF7L2, miR-Ctrl mimic, miR-Ctrl inhibitor, and siR-Ctrl were synthesized by GenePharma Corporation (Shanghai, China). The EX-TCF7L2 (pEZ-M02 vector), EX-Ctrl (pReceiver-M02 vector), wt-TCF7L2, mu-TCF7L2 were supplied by GeneCopoeia Corporation (Guangzhou, China). All sequences for transfection in this process are listed in Table 1.

\begin{tabular}{|c|c|c|}
\hline Genes & \multicolumn{2}{|c|}{ Sequence } \\
\hline miR-26b-5p mimic & & 5'-UUCAAGUAAUUCAGGAUAGGUdTdT-3 \\
\hline miR-Ctrl mimic & & 5'-UUGUACUACACAAAAGUACUGdTdT-3' \\
\hline miR-26b-5p inhibito & & 5'-ACCUAUCCUGAAUUACUUGAAdTdT-3' \\
\hline miR-Ctrl inhibitor & & 5'-CAGUACUUUUGUGUAGUACAAdTdT-3' \\
\hline \multirow[t]{2}{*}{ siTCF7L2 } & sense & 5'-GGGACAUGCAUGGAAUCAUTTdTdT-3 \\
\hline & Antisense & 5'-AUGAUUCCAUGCAUGUCCCTTdTdT-3' \\
\hline \multirow[t]{2}{*}{ siR-Ctrl } & sense & 5'-UUCUCCGAACGUGUCACGUdTdT-3' \\
\hline & antisense & 5-ACGUGACACGUUCGGAGAAdTdT-3 \\
\hline
\end{tabular}

Table 1. The constructed sequences used in this study

\section{Dual-Iuciferase reporter assay}

The synthesized sequence of TCF7L2 3' UTR-WT and TCF7L2 3'UTR-Mu were inserted between the Not I and Xho I cleavage sites of the psiCHECK-2 vector (Promega, Madison, WI, USA), downstream of the Renilla luciferase reporter gene. HEK-293 T cells were seeded into $96-$ well plates at 70\% confluence, and then co-transfected with each reporter construct (pmirGLO-TCF7L2-WT and pmirGLO-TCF7L2-Mu, and LvmiR-26b-5p, Lv-miR-NC, Lv-ASO-miR-26b-5p or LvASO-NC). After $48 \mathrm{~h}$, the Firefly and Renilla luciferase activities were detected using the Dual-Luciferase Reporter Gene Assay Kit (Beyotime, Shanghai, China). The Renilla luciferase was set as a reference to calculate the Firefly value. The sequences used in this part are listed in Table 1. 


\section{The effect of miR-26b-5p on TCF4 in hADMSCs by immunofluorescence}

miR-26b-5p mimic and inhibitor were transfected into the 4th-passage hADMSCs for detecting their effects of miR-26b-5p on TCF4 by immunofluorescence. Transfected hADMSCs were seeded on coverslips in 6-well plates and incubated at $37^{\circ} \mathrm{C}$ and $5 \% \mathrm{CO} 2$ for $48 \mathrm{~h}$. Then, hADMSCs were fixed by $4 \%$ paraformaldehyde for $15 \mathrm{~min}$, and then washed 3 times by PBS. 0.5\% Triton X-100 (Sigma-Aldrich, USA) was added to each well for $20 \mathrm{~min}$ at room temperature. After rinsing 3 times with phosphate-buffered saline (PBS), hADMSCs was blocked by goat serum for $2 \mathrm{~h}$. Primary antibodies specific for TCF-4 (1:1000, R\&D Systems, USA) were added to cells, and then incubated overnight at $4{ }^{\circ} \mathrm{C}$. Fluorescent Cy 3 fluorescent secondary antibodies (1:50, Proteintech, USA) were added and incubated for $1 \mathrm{~h}$ at $37^{\circ} \mathrm{C}$ in the dark after rising 3 times by PBS Tween-20. After that, the nucleus was re-stained by 4',6-diamidino-2phenylindole (DAPI, Sigma-Aldrich, USA). Cells were subsequently viewed by fluorescence microscopy (ZEISS, Oberkochen, Germany).

\section{Gene expression detection by qPCR}

Trizol Reagent was used to isolate total RNA of hADMSCs, and the purity of total RNA was detected by NanoVue ${ }^{\mathrm{Tm}}$ Plus spectrophotometer (GE Healthcare Life Sciences, USA) at 260/280 nm. cDNA was synthesized from RNA by RNA to cDNA EcoDry Premix (Double Primed)TM (TaKaRa, Japan) following the manufacturer's manual. qPCR was implemented by ABI 7300 Real-Time PCR System (Applied Biosystems, UK) using SYBR Premix ExTaq kit (TaKaRa, Japan). The following mRNA of genes associated with adipogenic differentiation and Wnt/ $\beta$-catenin pathway were detected in this study, recombinant human CCAAT/enhancer-binding protein a (C/EBPa), PPARY, adipocyte protein 2 (aP2), TCF7L2, $\beta$-catenin, and glyceraldehyde-3-phosphate dehydrogenase (GAPDH). The results of qPCR were calculated by the comparative delta-delta $\mathrm{Ct}$ method normalized with GAPDH. All primer sequences in this study were shown in Table 2 . 


\begin{tabular}{|c|c|c|}
\hline Gene & $\operatorname{Tm}\left({ }^{\circ} \mathrm{C}\right)$ & \\
\hline \multirow[t]{2}{*}{$\mathrm{C} / \mathrm{EBPa}$} & F: CTGATTCTTGCCAAACTGAG & 60 \\
\hline & R: GAGGAAGCTAAGACCCACTAC & 60 \\
\hline \multirow[t]{2}{*}{ PPARY } & F: CTTGACAGGAAAGACAACGG & 60 \\
\hline & R: GCTTCTACGGATCGAAACTG & 60 \\
\hline \multirow[t]{2}{*}{ aP2 } & F: AAATCACCGCAGACGACAGG & 60 \\
\hline & R: GGCTCATGCCCTTTCATAAAC & 60 \\
\hline \multirow[t]{2}{*}{ TCF7L2 } & F: CGGCGGTGGAGGGGATGAC & 60 \\
\hline & R: GGCCGCTTCTTCCAAACTTTCC & 60 \\
\hline \multirow[t]{2}{*}{$\beta$-catenin } & F: AAAATGGCAGTGCGTTTAG & 60 \\
\hline & R: TTTGAAGGCAGTCTGTCGTA & 60 \\
\hline \multirow[t]{2}{*}{ GAPDH } & F: GAACGGGAAGCTCACTGG & 60 \\
\hline & R: GCCTGCTTCACCACCTTCT & 60 \\
\hline
\end{tabular}

Table 2. The sequence of primer used for GPCR in this study

\section{Protein expression detection by western blot}

Total protein was isolated from hADMSCs by Cell lysis buffer for Western and IP kit (Beyotime, China) on ice. The concentrations of total protein were detected by BCA Protein Assay Kit (Beyotime, China) following the manufacture's manual. Protein with $5 \times$ SDS loading buffer (Beyotime, China) was denatured by $100^{\circ} \mathrm{C}$ for $5 \mathrm{~min}$. The electrophoresis of protein run in the SDS polyacrylamide gel electrophoresis at $100 \mathrm{~V}$ for $1.5 \mathrm{~h}$. After that, the protein blots were transferred onto membranes (GE Healthcare Life Sciences, USA). The membranes were blocked in TBS containing $5 \%$ non-fat milk at $37{ }^{\circ} \mathrm{C}$ for $2 \mathrm{~h}$. The proteins associated with adipogenic differentiation and $\mathrm{Wnt} / \beta$-catenin pathway were detected in this study, the protein and the concentration of their antibodies were listed below. C/EBPa (1:1000, Abcam, USA), PPARy (1:1000, Abcam, USA), aP2 (1:1000, Abcam, USA), TCF4 (1:1000, R\&D Systems, USA), $\beta$-catenin (1:1000, Abcam, USA) and GAPDH (1:1000, Cell Signaling Technology, USA). All primary antibodies mentioned above incubated with the membranes overnight at $4^{\circ} \mathrm{C}$. After that, unbound primary antibodies were washed away by TBS-0.05\% Tween 20 for 3 times. The secondary antibodies, which were specific binding to the primary antibodies mentioned above, were incubated with the membranes for $1 \mathrm{~h}$ at room temperature. The protein blots on membranes were developed by ECL solution (Thermo Fisher Scientific, Germany). Grayscale of blots and compared with GAPDH were analyzed by Quantity One (Bio-Rad, Hercules, CA, USA). 


\section{Statistical analysis}

The differences between multiple groups were analyzed by one-way analysis of variance (ANOVA). The differences between the two groups were analyzed by two-way ANOVA. SPSS 20.0 software (IBM SPSS Statistics, Armonk, NY: IBM-Corp.) was used to analyze the differences mentioned above, $p<0.05$ was set to the statistical significance.

\section{Results}

\section{The adipogenic differentiation of hADMSCs}

After cultured in the adipogenic medium at $37^{\circ} \mathrm{C}$ and $5 \% \mathrm{CO} 2$ for $4 \mathrm{~d}$, hADMSCs were harvested to detect the adipogenic differentiation and Wnt/ $\beta$-catenin pathway by the $\mathrm{qPCR}$ and western blot analysis. The group of hADMSCs set as control, which was cultured in a-MEM with $10 \% \mathrm{FBS}$ at $37^{\circ} \mathrm{C}$ and $5 \% \mathrm{CO} 2$.

The mRNAs associated with adipogenic differentiation (C/EBPa, PPARY and aP2) were significantly increased $9.13 \pm 3.96$-fold $(p=0.003), 51.01 \pm 16.96$-fold $(p=0.005)$ and $27.03 \pm 8.24$-fold $(p=0.019)$, respectively. The mRNAs associated with the Wnt/ $\beta$-catenin pathway (TCF7L2 and $\beta$-catenin) were significantly decreased $3.22 \pm 0.08$-fold $(p=0.003)$ and $4.78 \pm 0.07$-fold $(p=0.001)$, respectively (Fig. 2 . A).

The proteins associated with adipogenic differentiation (C/EBPa, PPARY and aP2) were significantly increased $1.72 \pm 0.17$-fold $(p=0.005), 2.76 \pm 0.26$-fold $(p<0.001)$ and $2.03 \pm 0.23$-fold $(p=0.001)$, respectively. The proteins associated with the Wnt/ $\beta$-catenin pathway (TCF4, cytoplasmic $\beta$-catenin and nuclear $\beta$-catenin) were significantly decreased $1.29 \pm 0.07$-fold $(p=0.019), 2.11 \pm 0.31$-fold $(p=0.002)$ and $1.77 \pm 0.21$-fold $(p=0.001)$, respectively (Fig. 2. B).

\section{TCF7L2 is target of miR-26b-5p}

The sequences of miR-26b-5p were highly conservative across species (Fig. 3. A), and it was likely matched to 3'-UTR of TCF7L2 (Fig. 3. B).

Comparing the control group, overexpression of miR-26b-5p significantly decreased the luciferase activity of pMIR/TCF7L2-WT reporter genes to $1.99 \pm 0.30$-fold $(p=0.002)$. Comparing the control group, overexpression of miR-26b-5p could not change the luciferase activity of reporter gene for TCF7L2 mRNA 3 '-UTR mutated. Comparing the control group, low expression of miR-26b-5p significantly increased the luciferase activity to $1.36 \pm 0.83$-fold $(p=0.036)$. Comparing the control group, low expression of miR-26b$5 p$ could not change the luciferase activity of reporter gene for TCF7L2 mRNA 3'-UTR mutated. The results were shown in Fig. 4. C and D.

Comparing to hADMSCs transfected with miR-Ctrl mimic, the expression level of TCF7L2 mRNA in hADMSCs, transfected with miR-26b-5p mimics, was decreased $5.00 \pm 0.64$-fold $(p=0.007)$ by qPCR, respectively. Comparing to hADMSCs transfected with miR-Ctrl inhibitor, the expression of TCF7L2 mRNA in hADMSCs, transfected with miR-26b-5p inhibitor, was increased $1.78 \pm 0.57$-fold $(p=0.014)$ by qPCR 
(Fig. 3. E). Comparing to hADMSCs transfected with miR-Ctrl mimic, the proteins of TCF4 expression in hADMSCs transfected with miR-26b-5p mimic were decreased $1.75 \pm 0.10$-fold $(p=0.004)$ by western blot. Comparing to hADMSCs transfected with miR-Ctrl inhibitor, TCF4 protein expression in hADMSCs transfected with miR-26b-5p inhibitor was increased $1.29 \pm 0.08$-fold $(p=0.020)$ by western blot, comparing to hADMSCs transfected with miR-Ctrl inhibitor (Fig. 3. F). Based upon all results in this part, TCF7L2 was target gene mediated by miR-26b-5p.

\section{The effect of miR-26b-5p on the immunofluorescence of TCF-4 and $\beta$-catenin in hADMSCs}

Comparing with normal cultured hADMSCs, the immunofluorescence intensity of TCF 4 and $\beta$-catenin were significantly inhibited by miR-26b-5p mimic (Fig. 4. A). Comparing with normal cultured hADMSCs, the immunofluorescence intensity of TCF4 and $\beta$-catenin were significantly promoted by miR-26b-5p inhibitor (Fig. 4. B).

\section{TCF-4 affects the adipogenic differentiation of hADMSCs}

After transfecting EX-TCF7L2, siTCF7L2, EX-Ctrl and siR-Ctrl to hADMSCs, the transfecting efficiency was detected by qPCR. After transfecting EX-TCF7L2 in hADMSCs, TCF7L2 expression increased $2.74 \pm 1.09$ fold $(p=0.011)$ than the EX-Ctrl group (Fig. 5. A). After transfecting siTCF7L2 in hADMSCs, TCF7L2 expression significantly decreased $3.81 \pm 0.54$-fold $(p=0.001)$ than the siR-Ctrl group (Fig. 5. A).

It was detected by qPCR analysis that the effect of TCF7L2 on the adipogenic differentiation of hADMSCs at the 4th d. After transfecting EX-TCF7L2 in hADMSCs, the expression of C/EBPa, PPARY, aP2 significantly respectively decreased $2.07 \pm 0.35$-fold $(p=0.012), 1.83 \pm 0.38$-fold $(p=0.002)$ and $3.23 \pm$ 1.38-fold ( $p=0.043)$; TCF7L2 and $\beta$-catenin significantly respectively increased $2.10 \pm 0.68$-fold $(p=$ 0.019 ) and $1.47 \pm 0.22$-fold ( $p=0.015$ ), than the EX-Ctrl group (Fig. 5. B). After transfecting siTCF7L2 in hADMSCs, the expression of C/EBPa, PPARy, aP2 significantly respectively increased $4.40 \pm 0.66$-fold ( $p$ $=0.043), 7.03 \pm 3.25$-fold $(p=0.034)$ and $4.04 \pm 0.62$-fold $(p<0.008)$; TCF7L2 and $\beta$-catenin significantly respectively decreased $5.11 \pm 1.52$-fold $(p=0.003)$ and $1.85 \pm 0.24$-fold $(p=0.037)$, than the siR-Ctrl group (Fig. 5. C).

So the adipogenic differentiation of hADMSCs was promoted by low-expression of TCF7L2.

\section{$\beta$-catenin affects the adipogenic differentiation of hADMSCs}

After transfecting EX- $\beta$-catenin, si $\beta$-catenin, EX-Ctrl and siR-Ctrl to hADMSCs, the transfecting efficiency was detected by qPCR. After transfecting EX- $\beta$-catenin in hADMSCs, $\beta$-catenin expression increased 2.92 \pm 0.84 -fold $(p=0.001$ ) than the EX-Ctrl group (Fig. 6. A). After transfecting si $\beta$-catenin in hADMSCs, $\beta$ catenin expression significantly decreased $3.22 \pm 0.55$-fold $(p=0.002)$ than the siR-Ctrl group (Fig. 6. A). 
It was detected by qPCR analysis that the effect of $\beta$-catenin on the adipogenic differentiation of hADMSCs at the 4th $d$. After transfecting EX- $\beta$-catenin in hADMSCs, the expression of C/EBPa, PPARY, aP2 significantly respectively decreased $2.36 \pm 0.78$-fold $(p=0.010), 4.32 \pm 0.98$-fold $(p=0.003)$ and 2.16 \pm 0.87 -fold $(p=0.010)$; TCF7L2 and $\beta$-catenin significantly respectively increased $3.18 \pm 0.69$-fold $(p=$ $0.002), 4.09 \pm 0.64$-fold $(p=0.002)$, than the EX-Ctrl group (Fig. 6. B). After transfecting si $\beta$-catenin in hADMSCs, the expression of C/EBPa, PPARY, aP2 significantly respectively increased $3.28 \pm 0.33$-fold ( $p$ $=0.001), 5.93 \pm 0.96$-fold $(p=0.013)$ and $4.78 \pm 1.54$-fold $(p=0.012)$; TCF7L2 and $\beta$-catenin significantly respectively decreased $2.61 \pm 0.54$-fold $(p=0.007)$ and $3.67 \pm 1.73$-fold $(p=0.008)$, than the siR-Ctrl group (Fig. 6. C).

Based upon the results above, the adipogenic differentiation of hADMSCs was promoted by the lowexpression of $\beta$-catenin.

\section{Effects of miR-26b-5p on adipogenic differentiation of hADMSCs}

After transfecting miR-26b-5p mimic, inhibitor, miR-Ctrl mimic, and miR-Ctrl inhibitor in hADMSCs, the transfecting efficiency were detected by qPCR. After transfecting miR-26b-5p mimic in hADMSCs, the expression of miR-26b-5p increased $161.15 \pm 41.26$-fold $(p<0.001)$ than the miR-Ctrl mimic group (Fig. 7 . A). After transfecting miR-26b-5p inhibitor in hADMSCs, the expression of miR-26b-5p decreased $3.88 \pm$ 0.20 -fold $(p=0.012)$ than the miR-Ctrl inhibitor group (Fig. 7. A).

It was detected by qPCR analysis that the effect of miR-26b-5p on the adipogenic differentiation of hADMSCs at the 4th $d$. After transfecting miR-26b-5p mimic in hADMSCs, the expression of C/EBPa, PPARy, aP2 significantly respectively increased $5.83 \pm 1.99$-fold $(p=0.029), 3.99 \pm 3.14$-fold $(p=0.002)$ and 10.17 \pm 4.81 -fold $(p=0.005)$; TCF7L2 and $\beta$-catenin significantly respectively decreased $3.80 \pm 1.96$ fold $(p=0.006)$ and $2.80 \pm 1.34$-fold $(p=0.016)$, than the miR-Ctrl mimic group (Fig. 7. B). After transfecting miR-26b-5p inhibitor in hADMSCs, the expression of C/EBPa, PPARy, aP2 significantly respectively decreased $1.64 \pm 0.47$-fold $(p=0.026), 2.12 \pm 0.71$-fold $(p=0.009)$ and $2.31 \pm 0.72$-fold $(p=$ $0.039)$; TCF7L and $\beta$-catenin significantly respectively increased $2.13 \pm 0.36$-fold $(p=0.003)$ and $1.84 \pm$ 0.28 -fold $(p=0.003)$, than the miR-Ctrl inhibitor group (Fig. 7. C).

Based upon the results above, the adipogenic differentiation of hADMSCs was promoted by overexpression of miR-26b-5p.

\section{TCF4 regulated by miR-26b-5p influencing the adipogenic differentiation of hADMSCs}

After cotransfecting with miR-26b-5p mimic and EX-TCF7L2 in hADMSCs, the mRNA expression levels of C/EBPa, PPARY and aP2 significantly respectively increased $2.69 \pm 0.10$-fold ( $p=0.007$ ), $3.58 \pm 1.47$-fold $(p=0.002)$ and $1.75 \pm 0.55$-fold $(p=0.013)$; TCF7L2 and $\beta$-catenin significantly respectively decreased $2.13 \pm 0.99$-fold $(p=0.014), 2.65 \pm 0.31$-fold $(p=0.006)$, than those in cotransfecting with miR-26b-5p 
mimic and EX-Ctrl (Fig. 8. A). The protein expression levels of C/EBPa, PPARY and aP2 significantly respectively increased $1.28 \pm 0.08$-fold $(p=0.048), 1.79 \pm 0.21$-fold $(p=0.004)$ and $1.30 \pm 0.19$-fold $(p=$ $0.025)$; TCF4, cytoplasmic $\beta$-catenin and nuclear $\beta$-catenin significantly respectively decreased $1.47 \pm$ 0.09 -fold $(p=0.003), 1.55 \pm 0.03$-fold $(p=0.015)$ and $1.29 \pm 0.07$-fold $(p=0.042)$, than those in cotransfecting with miR-26b-5p mimic and EX-Ctrl (Fig. 8. B). The immunofluorescence intensity of $\beta$ catenin in the hASC nucleus was also increased in hADMSCs cotransfected with the miR-26b-5p mimic and EX-TCF7L2 (Fig. 8. C).

After cotransfecting with miR-26b-5p inhibitor and siTCF7L2 in hADMSCs, the mRNA levels of C/EBPa, PPARy and aP2 significantly respectively decreased $2.56 \pm 1.23$-fold $(p=0.013), 2.51 \pm 0.94$-fold $(p=$ $0.031)$ and $1.60 \pm 0.54$-fold ( $p=0.023)$; TCF4 and $\beta$-catenin significantly respectively increased $2.20 \pm$ 0.09 -fold $(p=0.017)$ and $1.95 \pm 0.46$-fold $(p=0.010)$, than those in miR-26b-5p inhibitor and siR-Ctrl (Fig. 8. D). The protein expression levels of C/EBPa, PPARY and aP2 significantly respectively decreased $1.43 \pm 0.21$-fold $(p=0.008), 1.68 \pm 0.05$-fold $(p=0.004)$ and $1.37 \pm 0.13$-fold $(p=0.008)$; TCF4, cytoplasmic $\beta$-catenin and nuclear $\beta$-catenin significantly respectively increased $1.29 \pm 0.14$-fold $(p=$ $0.039), 1.44 \pm 0.20$-fold $(p=0.028)$ and $1.25 \pm 0.16$-fold $(p=0.025)$, than those in miR-26b-5p inhibitor and siR-Ctrl (Fig. 8. E). After cotransfected with the miR-26b-5p inhibitor and siTCF7L2 in hADMSCs, the immunofluorescence intensity of $\beta$-catenin both in the hASC cytoplasma and nucleus was also decreased in hADMSCs (Fig. 8. F).

\section{$\beta$-catenin regulated by miR-26b-5p influencing the adipogenic differentiation of hADMSCs}

After cotransfecting with miR-26b-5p mimic and EX- $\beta$-catenin in hADMSCs, the mRNA expression levels of C/EBPa, PPARY and aP2 significantly respectively increased $4.62 \pm 1.78$-fold $(p=0.004), 2.73 \pm 0.37$ fold $(p=0.007)$ and $4.64 \pm 2.16$-fold $(p=0.001)$; TCF7L2 and $\beta$-catenin significantly respectively decreased $1.97 \pm 0.82$-fold ( $p=0.019), 3.30 \pm 0.52$-fold $(p=0.036)$, than those in cotransfecting with miR26b-5p mimic and EX-Ctrl (Fig. 9. A). The protein expression levels of C/EBPa, PPARY and aP2 significantly respectively increased $1.45 \pm 0.07$-fold $(p=0.027), 1.53 \pm 0.10$-fold $(p=0.013)$ and $1.36 \pm$ 0.14 -fold $(p=0.040)$; TCF4, cytoplasmic $\beta$-catenin and nuclear $\beta$-catenin significantly respectively decreased $1.37 \pm 0.19$-fold $(p=0.014), 1.45 \pm 0.16$-fold $(p=0.041)$ and $1.39 \pm 0.12$-fold $(p=0.018)$, than those in cotransfecting with miR-26b-5p mimic and EX-Ctrl (Fig. 9. B). The immunofluorescence intensity of $\beta$-catenin in the hASC nucleus was also decreased in hADMSCs cotransfected with the miR-26b-5p mimic and EX- $\beta$-catenin (Fig. 9. C). miR-26b-5p mimic partially reversed the inhibitory effect of $\beta$-catenin on adipogenic differentiation of hADMSCs.

After cotransfecting with miR-26b-5p inhibitor and siß-catenin in hADMSCs, the mRNA levels of C/EBPa, PPARY and aP2 significantly respectively decreased $1.56 \pm 0.17$-fold $(p=0.030), 1.78 \pm 0.37$-fold $(p=$ $0.005)$ and $2.76 \pm 0.69$-fold $(p=0.001)$;

TCF7L2 and $\beta$-catenin significantly respectively increased $1.67 \pm 0.31$-fold $(p=0.013)$ and $1.69 \pm 0.51$-fold $(p=0.024)$, than those in miR-26b-5p inhibitor and siR-Ctrl (Fig. 9. D). The protein expression levels of 
C/EBPa, PPARy and aP2 significantly respectively decreased $1.30 \pm 0.05$-fold $(p=0.005), 1.29 \pm 0.03$-fold $(p=0.017)$ and $1.85 \pm 0.02$-fold $(p=0.001)$; TCF4, cytoplasmic $\beta$-catenin and nuclear $\beta$-catenin significantly respectively increased $1.39 \pm 0.07$-fold $(p=0.026), 1.30 \pm 0.08$-fold $(p=0.018)$ and $1.66 \pm$ 0.09-fold ( $p=0.009$ ), than those in miR-26b-5p inhibitor and siR-Ctrl (Fig. 9. E). After cotransfected with the miR-26b-5p inhibitor and si $\beta$-catenin in hADMSCs, the immunofluorescence intensity of $\beta$-catenin both in the hASC cytoplasma and nucleus was also decreased in hADMSCs (Fig. 9. F).

Based upon the results above, the stimulative effect of $\beta$-catenin low-expression in adipogenic differentiation was inhibited by of miR-26b-5p inhibitor.

\section{Discussion}

Adipose tissue is abundant and extensive in the body, and they have a sufficient tissue supply to repair soft tissue defects[9]. Coleman introduced a procedure based on a methodology and the use of specific material for adipose filling[15, 16]. However, autologous adipose tissue may lose their volume of up to $60 \%$ after transplantation because of necrosis $[17,18]$. So, it is hard to achieve the goal of soft tissue augmentation.

How to reduce the absorption of fat tissue is the crucial point of fat tissue transplantation. The transplantation of ADSMCs mixed with fat particles can effectively reduce the absorption of transplanted fat $[19,20]$. The softer texture of transplanted adipose tissue with mixed ADSMCs; a fat structure remains intact; fat lobules and leaflet intervals are kept well. ADSMCs secrete a variety of pro-angiogenic cytokines, such as VEGF, bFGF, HGF, IGF-1, etc, which could promote blood supply and early graft survival $[21,22]$. The addition of ADMSCs to fat granules for transplantation has a higher survival rate than single fat granule transplantation $[23,24]$.

Although ADSMCs could retain the volume of transplanted adipose tissue, there were some potential risks for postoperative soft tissue restoration in patients with malignant tumors. At present, there is no sufficient evidence that ASCs have a potential carcinogenic effect. But in many studies proved that ADMSCs could secrete VEGF,C-Kit, PDGF could promote endothelial proliferation and neoangiogenesis, and supporting tumor growth and metastasis in many types of malignant tumors $[25,26]$. In clinical, autologous fat graft mainly used in the repair and treatment of breast cancer. The American Society of Plastic Surgeons illustrated a level-B recommendation show that fat grafting transplantation does not increase the risk of local recurrence (https://www.plasticsurgery.org/Documents/Health-

Policy/Principles/principle-2015-post-mastectomy-fat-grafting.pdf). Nevertheless, the clinical application of this technique to repair patients with soft tissue defects who underwent malignant tumors surgery requires further research.

Wnt signaling pathway plays an inhibition role in adipogenic differentiation of MSCs [27]. 3T3-L1 preadipocytes were maintained in an undifferentiated state by increased expression of Wnt10b, which inhibited the expression of PPARY and C/EBP-a $[28,29]$. The activation of Wnt/ $\beta$-catenin pathway via the ectopic expression of Wnt1 and Wnt3a will prevent the adipogenic differentiation of MSCs by 
suppression of PPARY [27, 30,31]. Wnt10a and Wnt6 as endogenous regulators of adipogenesis and overexpression of Wnt6 and Wnt10a blocked adipogenesis [32]. The overexpression fo PPARy may inhibit the activity of Wnt/ $\beta$-catenin pathway, but overexpression of PPARy and/or C/EBPa could not rescue Wnt/ $\beta$-catenin-mediated inhibition of adipogenesis[33, 34].

Some scholars have shown that the change of TCF-4 expression plays an important regulatory role in adipogenic differentiation. Singh R. reported that during the process of 3T3-L1 cells differentiated into adipocytes in adipogenic medium, transfection of TCF4 CDNA inhibited adipogenic differentiation[35]. Zhang et al. reported that high-glucose conditions inhibited the nuclear translocation of $\beta$-catenin/TCF- 4 and promoted adipogenesis in cardiac stem cells [36]. Embelin has the potential to prevent body weight gain; after Embelin treated, both the proliferation and adipogenic differentiation were inhibited in ST2 and $\mathrm{C} 3 \mathrm{H} 10 \mathrm{~T} 1 / 2$ cells, nuclear protein levels of $\beta$-catenin and TCF-4 were increased [37]. Myostatin enhanced nuclear translocation of $\beta$-catenin and formation of the Smad3- $\beta$-catenin-TCF4 complex, together with the altered expression of a number of Wnt/ $\beta$-catenin pathway genes in hMSCs. The inhibitory effects of myostatin on adipogenesis were blocked by RNAi silencing of $\beta$-catenin and diminished by overexpression of dominant-negative TCF4 [38]. Overexpression of dominant-negative TCF4 inactivated Wnt signaling pathway in preadipocytes; on the contrary, these cells differentiate into adipocytes [27].

Mechanistic investigation revealed that the overexpression of miR-139 suppressed the $\beta$-catenin/TCF- 4 transcriptional activity by targeting TCF-4 in hepatocellular carcinoma [39]. Zhou et al. indicated that miR212 inhibited cervical cancer metastasis and progression by targeting TCF7L2 expression [40]. TCF-4 was a direct target of miR-379 in laryngeal carcinoma; overexpression of miR-379 inhibited cell proliferation, migration, and invasion [41]. Tian et al. reported that Tcf7l2 is a miR-17-5p target and confirmed the repressive effect of Tcf7I2 on 3T3-L1 adipogenic differentiation [42]. Li et al. .reported that the expression of miR-328 inhibited the proliferation of cervical cancer cells by targeting Tcf7l2 [43].

Existing research indicates that $\mathrm{miR}-26 \mathrm{~b}-3 \mathrm{p}$ is involved in the regulation of a variety of biological processes. miR-26b-3p was significantly upregulated during serial in vitro passage of human umbilical cord-derived mesenchymal stem cells and was correlated with cellular senescence and cell cycle genes; overexpression of miR-26b-3p inhibited the proliferation of these cells in vitro [44]. Lin reported that miR26b-3p suppresses osteoblast differentiation of MC3T3-E1 cells via directly targeting estrogen receptor a [45]. miR-26b-3p was significantly upregulated in whole blood in patients with rheumatoid arthritis and their asymptomatic first-degree relatives [46]. Ginsenoside Rh2 inhibited the expression of miR-26b-3p in liver cancer cells [47]. miR-26b-3p were upregulated in patients with Alzheimer's disease than normal people [48].

\section{Conclusions}

Suppressive expression of miR-26b-5p and low-expression of TCF-4 promoting the adipogenic differentiation of hADMSCs in vitro. miR-26b-5p is a negative regulator to inhibit TCF-4 directly, and then inactivated Wnt/ $\beta$-catenin pathway, which promotes the adipogenic differentiation of hADMSCs in vitro. 


\section{Abbreviations}

hADMSCs

human adipose-derived mesenchymal stem cells

miRNAs

MicroRNAs

PPARY

peroxisome proliferator-activated receptory

TCF-4

$\mathrm{T}$ cell factor 4

a-MEM

Alpha-modified Eagle's medium

FBS

fetal bovine serum

qPCR

Real-time Quantitative PCR

SDS

Sodium dodecyl sulfate

EX-TCF7L2

TCF7L2 expression plasmid

SiTCF7L2

TCF7L2 SIRNA

EX-Ctrl

Negative control expression plasmid

siR-Ctrl

Negative control siRNA

miR-Ctrl

Mimic negative control of the miR-26b-5p mimic

miR-Ctrl

Inhibitor negative control of the miR-26b-5p inhibitor

wt-TCF7L2

TCF7L2 3'UTR-wild type

mu-TCF7L2

TCF7L2 3'UTR-mutant

PBS

Phosphate-buffered saline

DAPI

4',6-diamidino-2-phenylindole

$\mathrm{C} / \mathrm{EBPa}$

recombinant human CCAAT/enhancer binding proteina 
aP2

adipocyte protein 2

GAPDH

Glyceraldehyde-3-phosphate dehydrogenase

ANOVA

Analysis of variance

\section{Declarations}

\section{Availability of data and materials}

The datasets generated and/or analyzed during the current study are not publicly available due but are available from the corresponding author upon reasonable request.

\section{Acknowledgements}

All authors are acknowledged for their contribution to the study.

\section{Funding}

Our work was funded by the National Natural Science Foundation of China, grant agreement number 81600908 and 81701491; and the Natural Science Foundation of Jiangsu Province, grant agreement number BK20170152.

\section{Contributions}

$H D, J M, X D, H W, Y C$ and $Y L$ were designed these experiment and literature research. $Y L, Y C, M L, Y M, H J$, and HS performed all procedures of experiments. SL, XD, JM and CW were in charge of the data analysisa and interpretation. The manuscript prepared by HD, YL, JH, JM and HW. All authors approval of the final version.

\section{Authors' information}

Not applicable.

\section{Corresponding author}

Correspondence to Hongming Du and Jian Meng.

\section{Ethics declarations}

\section{Ethics approval and consent to participate}

This study was approved by the ethics committee of the Nanjing Medical University; And all patients for fat tissue donors signed informed consent. 


\section{Consent for publication}

Not applicable.

\section{Competing interests}

The authors declare that they have no competing interests.

\section{References}

[1] Bradford BD, Lee JW. Reconstruction of the Forehead and Scalp. Facial Plast Surg Clin North Am. 2019;27(1):85-94.

[2] Saban Y. Rhinoplasty: lessons from "errors" : From anatomy and experience to the concept of sequential primary rhinoplasty. HNO. 2018;66(1):15-25.

[3] Kim RY, Sokoya M, Ducic Y, Williams F. Free-Flap Reconstruction of the Mandible.Semin Plast Surg. 2019;33(1):46-53.

[4] Stephan S, Reinisch J. Auricular Reconstruction Using Porous Polyethylene Implant Technique. Facial Plast Surg Clin North Am. 2018;26(1):69-85.

[5] Denadai R, Raposo-Amaral CA, Raposo-Amaral CE. Fat Grafting in Managing Craniofacial Deformities. Plast Reconstr Surg. 2019;143(5):1447-55.

[6] Borrelli MR, Hu MS, Longaker MT, Lorenz HP. Tissue Engineering and Regenerative Medicine in Craniofacial Reconstruction and Facial Aesthetics. J Craniofac Surg. 2020;31(1):15-27.

[7] Wang J, Liu S, Li J, Zhao S, Yi Z. Roles for miRNAs in osteogenic differentiation of bone marrow mesenchymal stem cells. Stem Cell Res Ther. 2019;10(1):197.

[8] Zuk PA. The adipose-derived stem cell: looking back and looking ahead. Mol Biol Cell. 2010;21(11):1783-7.

[9] Nguyen PN, Huang CJ, Sugii S, Cheong SK, Choo KB. Selective activation of miRNAs of the primatespecific chromosome 19 miRNA cluster (C19MC) in cancer and stem cells and possible contribution to regulation of apoptosis. J Biomed Sci. 2017;24(1):20.

[10] Ke J, Xu HE, Williams BO. Lipid modification in Wnt structure and function. Curr Opin Lipidol. 2013;24(2):129-33.

[11] Ke J, Xu HE, Williams BO. Lipid modification in Wnt structure and function. Curr Opin Lipidol. 2013;24(2):129-33. 
[12] Shi C, Huang F, Gu X, Zhang M, Wen J, Wang X, et al. Adipogenic miRNA and meta-signature miRNAs involved in human adipocyte differentiation and obesity. Oncotarget. 2016;7(26):40830-45.

[13] Tian L, Song Z, Shao W, Du WW, Zhao LR, Zeng K, et al.Curcumin represses mouse 3T3-L1 cell adipogenic differentiation via inhibiting miR-17-5p and stimulating the Wnt signalling pathway effector Tcf7l2. Cell Death Dis. 2017;8(1):e2559.

[14] Luo Y, Ge R, Wu H, Ding X, Song H, Ji H, et al. The osteogenic differentiation of human adiposederived stem cells is regulated through the let-7i-3p/LEF1/ $\beta$-catenin axis under cyclic strain. Stem Cell Res Ther. 2019;10(1):339.

[15] Coleman SR, Katzel EB. Fat Grafting for Facial Filling and Regeneration. Clin Plast Surg. 2015;42(3):289-300,

[16] Coleman SR. Facial recontouring with lipostructure. Clin Plast Surg. 1997;24(2):347-67.

[17] Silva ABD, Haupenthal F, Morais AD, Ascenço ASK, Sebastião APM, Cavalcanti MAR, et al. Relationship between Tamoxifen and the Absorption of Subfascial Autologous Fat Grafts. Plast Reconstr Surg. 2018;141(6):1408-15.

[18] Kim DY, Ji YH, Kim DW, Dhong ES, Yoon ES. Effects of platelet-rich plasma, adipose-derived stem cells, and stromal vascular fraction on the survival of human transplanted adipose tissue. J Korean Med Sci. 2014;29 Suppl 3:S193-200.

[19] Ha KY, Park H, Park SH, Lee BI, Ji YH, Kim TY, et al. The Relationship of a Combination of Human Adipose Tissue-Derived Stem Cells and Frozen Fat with the Survival Rate of Transplanted Fat. Arch Plast Surg. 2015;42(6):677-85

[20] Lu F, Li J, Gao J, Ogawa R, Ou C, Yang B, Fu B. Improvement of the survival of human autologous fat transplantation by using VEGF-transfected adipose-derived stem cells. Plast Reconstr Surg. 2009;124(5):1437-46.

[21] Bianchi F, Maioli M, Leonardi E, Olivi E, Pasquinelli G, Valente S, et al. A new nonenzymatic method and device to obtain a fat tissue derivative highly enriched in pericyte-like elements by mild mechanical forces from human lipoaspirates. Cell Transplant. 2013;22(11):2063-77.

[22] Zhu M, Zhou Z, Chen Y, Schreiber R, Ransom JT, Fraser JK, et al. Supplementation of fat grafts with adipose-derived regenerative cells improves long-term graft retention. Ann Plast Surg. 2010;64(2):222-8.

[23] Matsumoto D, Sato K, Gonda K, Takaki Y, Shigeura T, Sato T, et al. Cell-assisted lipotransfer: supportive use of human adipose-derived cells for soft tissue augmentation with lipoinjection. Tissue Eng. 2006;12(12):3375-82. 
[24] Yoshimura K, Sato K, Aoi N, Kurita M, Inoue K, Suga H, et al. Cell-assisted lipotransfer for facial lipoatrophy: efficacy of clinical use of adipose-derived stem cells. Dermatol Surg. 2008;34(9):1178-85.

[25] Salha S, Gehmert S, Brébant V, Anker A, Loibl M, Prantl L, et al. PDGF regulated migration of mesenchymal stem cells towards malignancy acts via the PI3K signaling pathway. Clin Hemorheol Microcirc. 2018;70(4):543-551.

[26] Preisner F, Leimer U, Sandmann S, Zoernig I, Germann G, Koellensperger E. Impact of Human Adipose Tissue-Derived Stem Cells on Malignant Melanoma Cells in An In Vitro Co-culture Model.

Stem Cell Rev Rep. 2018;14(1):125-40.

[27] Ross SE, Hemati N, Longo KA, Bennett CN, Lucas PC, Erickson RL, et al. Inhibition of adipogenesis by Wnt signaling. Science. 2000;289(5481):950-3.

[28] Liu J, Farmer SR. Regulating the balance between peroxisome proliferator-activated receptor gamma and beta-catenin signaling during adipogenesis. A glycogen synthase kinase 3beta phosphorylationdefective mutant of beta-catenin inhibits expression of a subset of adipogenic genes. J Biol Chem. 2004;279(43):45020-7.

[29] Moldes M, Zuo Y, Morrison RF, Silva D, Park BH, Liu J, et al. Peroxisome-proliferator-activated receptor gamma suppresses Wnt/beta-catenin signalling during adipogenesis. Biochem J. 2003;376(Pt 3):607-13.

[30] Liu J, Farmer SR. Regulating the balance between peroxisome proliferator-activated receptor gamma and beta-catenin signaling during adipogenesis. A glycogen synthase kinase 3beta phosphorylationdefective mutant of beta-catenin inhibits expression of a subset of adipogenic genes. J Biol Chem. 2004;279(43):45020-7.

[31] Lin GL, Hankenson KD. Integration of BMP, Wnt, and notch signaling pathways in osteoblast differentiation. J Cell Biochem. 2011;112(12):3491-501.

[32] Cawthorn WP, Bree AJ, Yao Y, Du B, Hemati N, Martinez-Santibañez G, et al. Wnt6, Wnt10a and Wnt10b inhibit adipogenesis and stimulate osteoblastogenesis through a $\beta$-catenin-dependent mechanism. Bone. 2012;50(2):477-89.

[33] Muruganandan S, Roman AA, Sinal CJ. Adipocyte differentiation of bone marrow-derived mesenchymal stem cells: cross talk with the osteoblastogenic program. Cell Mol Life Sci. 2009;66(2):23653.

[34] Kawai M, Mushiake S, Bessho K, Murakami M, Namba N, Kokubu C, et al. Wnt/Lrp/beta-catenin signaling suppresses adipogenesis by inhibiting mutual activation of PPARgamma and C/EBPalpha. Biochem Biophys Res Commun. 2007;363(2):276-82. 
[35] Singh R, Artaza JN, Taylor WE, Braga M, Yuan X, Gonzalez-Cadavid NF, et al. Testosterone inhibits adipogenic differentiation in 3T3-L1 cells: nuclear translocation of androgen receptor complex with betacatenin and T-cell factor 4 may bypass canonical Wnt signaling to down-regulate adipogenic transcription factors. Endocrinology. 2006;147(1):141-54.

[36] Zhang X, Meng K, Pu Y, Wang C, Chen Y, Wang L. Hyperglycemia Altered the Fate of Cardiac Stem Cells to Adipogenesis through Inhibiting the $\beta$-Catenin/TCF-4 Pathway. Cell Physiol Biochem. 2018;49(6):2254-63.

[37] Gao Y, Li J, Xu X, Wang S, Yang Y, Zhou J, et al. Embelin attenuates adipogenesis and lipogenesis through activating canonical Wnt signaling and inhibits high-fat diet-induced obesity. Int J Obes (Lond). 2017;41(5):729-738.

[38] Guo W, Flanagan J, Jasuja R, Kirkland J, Jiang L, Bhasin S. The effects of myostatin on adipogenic differentiation of human bone marrow-derived mesenchymal stem cells are mediated through crosscommunication between Smad3 and Wnt/beta-catenin signaling pathways. J Biol Chem. 2008;283(14):9136-45.

[39] Gu W, Li X, Wang J. miR-139 regulates the proliferation and invasion of hepatocellular carcinoma through the WNT/TCF-4 pathway. Oncol Rep. 2014;31(1):397-404.

[40] Zhou C, Tan DM, Chen L, Xu XY, Sun CC, Zong LJ, et al. Effect of miR-212 targeting TCF7L2 on the proliferation and metastasis of cervical cancer. Eur Rev Med Pharmacol Sci. 2017;21(2):219-226.

[41] Wei JL, Zhang L, Zhao ZM, Zhao YZ, Fu Q, Yang Y. MicroRNA-379 inhibits laryngeal carcinoma cell proliferation and invasion through directly targeting TCF-4. Kaohsiung J Med Sci. 2019;35(12):731-738.

[42] Tian L, Song Z, Shao W1, Du WW, Zhao LR, Zeng K, Yang BB, Jin T. Curcumin represses mouse 3T3L1 cell adipogenic differentiation via inhibiting miR-17-5p and stimulating the Wnt signalling pathway effector Tcf7l2. Cell Death Dis. 2017;8(1):e2559.

[43] Wang X, Xia Y. microRNA-328 inhibits cervical cancer cell proliferation and tumorigenesis by targeting TCF7L2. Biochem Biophys Res Commun. 2016;475(2):169-75.

[44] Wang Q, Xu C, Zhao Y, Xu Z, Zhang Y, Jiang J, et al. miR-26b-3p Regulates Human Umbilical CordDerived Mesenchymal Stem Cell Proliferation by Targeting Estrogen Receptor. Stem Cells Dev. 2016;25(5):415-26.

[45] Lin Y, Xiao L, Zhang Y, Li P, Wu Y, Lin Y. MiR-26b-3p regulates osteoblast differentiation via targeting estrogen receptor a. Genomics. 2019;111(5):1089-96.

[46] Anaparti V, Smolik I, Meng X, Spicer V, Mookherjee N, El-Gabalawy H. Whole blood microRNA expression pattern differentiates patients with rheumatoid arthritis, their seropositive first-degree relatives, and healthy unrelated control subjects. Arthritis Res Ther. 2017;19(1):249. 
[47] Chen W, Chu S, Li H, Qiu Y. MicroRNA-146a-5p enhances ginsenoside Rh2-induced anti-proliferation and the apoptosis of the human liver cancer cell line HepG2. Oncol Lett. 2018;16(4):5367-74.

[48] Satoh J, Kino Y, Niida S. MicroRNA-Seq Data Analysis Pipeline to Identify Blood Biomarkers for Alzheimer's Disease from Public Data. Biomark Insights. 2015;10:21-31.

\section{Figures}



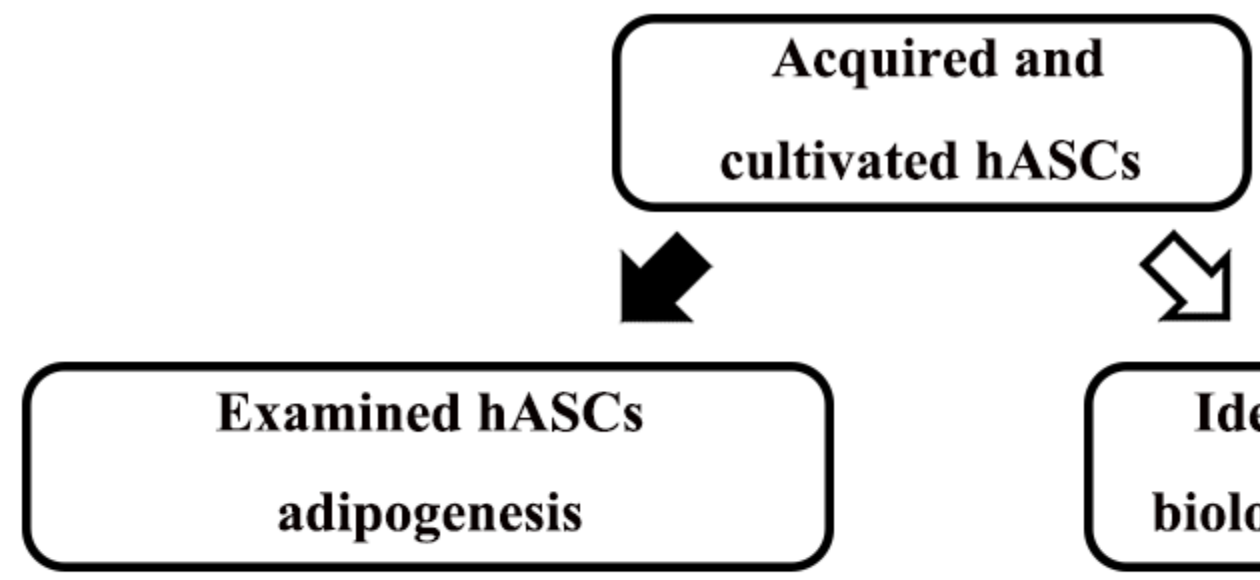

\section{Identified hASCs biological characters}

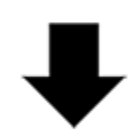

Microarray analysis proved the low expression of miR-26b-5p

Promoted and suppressed of

TCF4 and $\beta$-catenin \& examined related genes

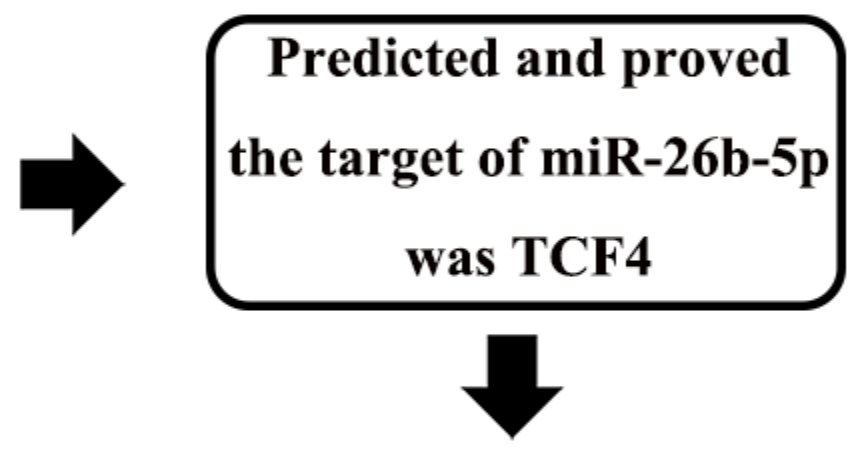

\section{Promoted and suppressed of miR-26b-5p \& examined \\ of related genes}
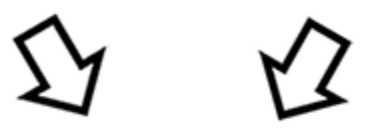

\section{Promoted and suppressed of} miR-26b-5p to compensate TCF4 effects \& examine related genes

\section{Figure 1}

\section{Experimental procedure in this study.}



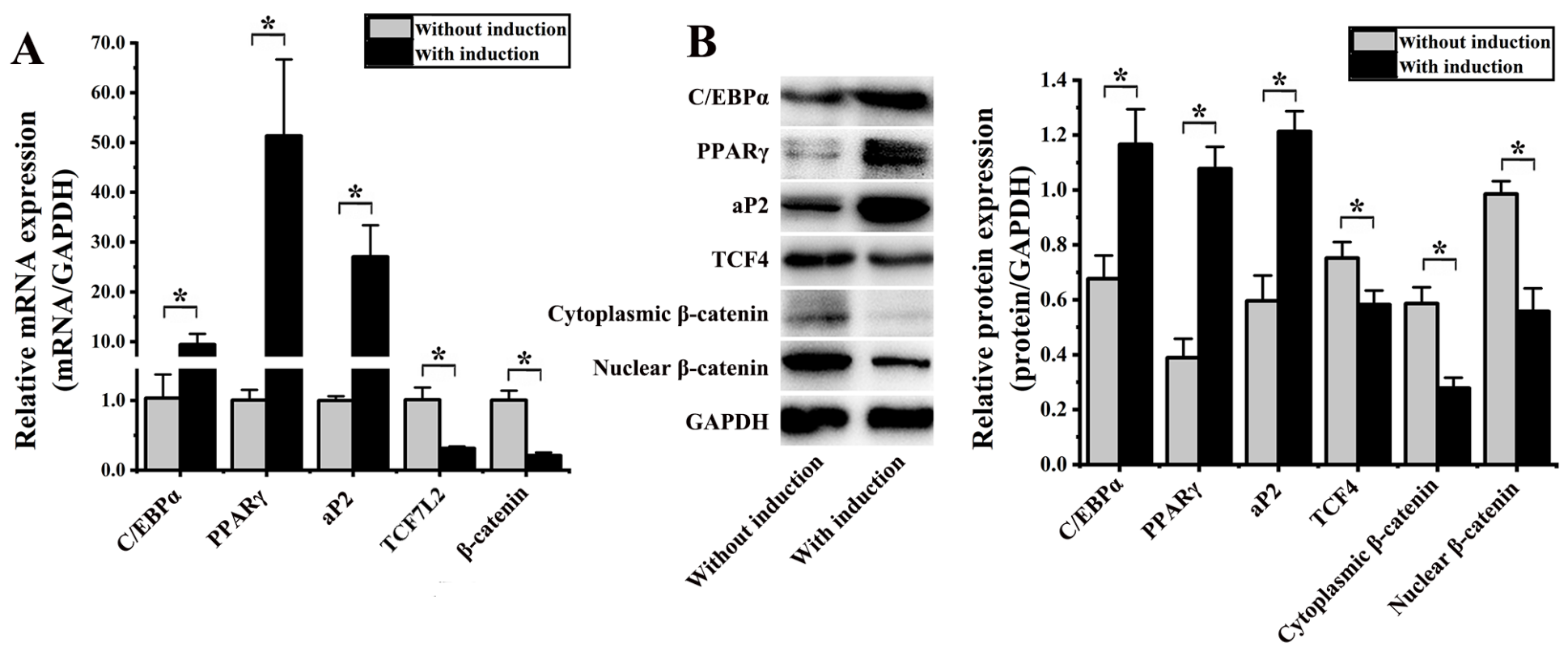

Figure 2

The osteogenic differentiation of hADMSCs under cyclic strain. $\left({ }^{\star} \mathrm{p}<0.05\right.$, there were significant difference between these two groups) (A) Results of qPCR. The following mRNAs were increased significantly: C/EBP a $9.13 \pm 3.96$-fold $(p=0.003)$, PPARy 51.01 \pm 16.96 -fold $(p=0.005)$, aP2 $27.03 \pm$ 8.24-fold ( $p=0.019)$. The following mRNAs were decreased significantly: TCF7L2 $3.22 \pm 0.08$-fold ( $p=$ $0.003)$, $\beta$-catenin $4.78 \pm 0.07$-fold $(p=0.001)$. (B) Results of western blot. Protein blots were listed in left column. The following proteins were increased significantly: C/EBP a $1.72 \pm 0.17$-fold ( $p=0.005)$, PPARY $2.76 \pm 0.26$-fold $(p<0.001)$, aP2 $2.03 \pm 0.23$-fold $(p=0.001)$. The following proteins were decreased significantly: TCF4 $1.29 \pm 0.07$-fold $(p=0.019)$, cytoplasmic $\beta$-catenin $2.11 \pm 0.31$-fold $(p=0.002)$, nuclear $\beta$-catenin $1.77 \pm 0.21$-fold $(p=0.001)$. 
A

Sequences of miR-26b-5p among species

\begin{tabular}{|c|c|}
\hline Species & Sequences of miR-26b-5p \\
\hline Homo sapiens & 5'... UUCAAGUAAUUCAGGAUAGGU... 3' \\
\hline Gadus morhua & ... UUCAAGUAAUCCAGGAUAGGUU... \\
\hline Dasypus novemcinctus & ... UUCAAGUAAUUCAGGAUAGGUU... \\
\hline Sus scrofa & $\begin{array}{l}\text {... UUCAAGUAAUUCAGGAUAGGUU... } \\
\text { wt-TCF7L2 }\end{array}$ \\
\hline Macaca mulatta & ... UUCAAGUAAUUCAGGAUAGGU... \\
\hline Cavia porcellus & ... UUCAAGUAAUUCAGGAUAGGUU... \\
\hline Pteropus alecto & ... UUCAAGUAAUUCAGGAUAGGUU... \\
\hline Oryctolagus cuniculus & ... UUCAAGUAAUUCAGGAUAGGUU... \\
\hline Capra hircus & ... UUCAAGUAAUUCAGGAUAGGUU... \\
\hline \multicolumn{2}{|c|}{ B Predicted consequential pairing of miRNA and target region } \\
\hline Hsa-miR-26-5p & UGGAUAGGACUUAAUGAACUU \\
\hline $\begin{array}{l}\text { Position 3716-3722 of } \\
\text { TCF7L2 3' UTR }\end{array}$ & 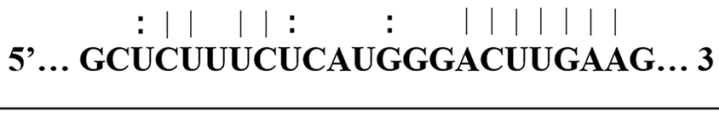 \\
\hline
\end{tabular}
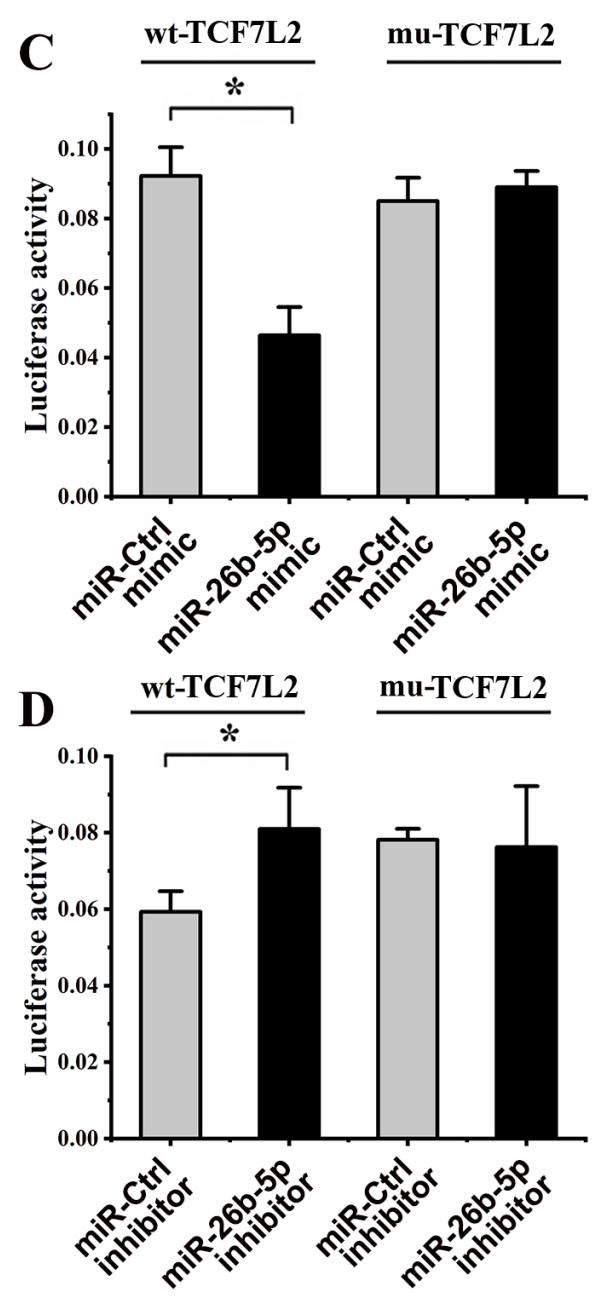

$\mathbf{E}$

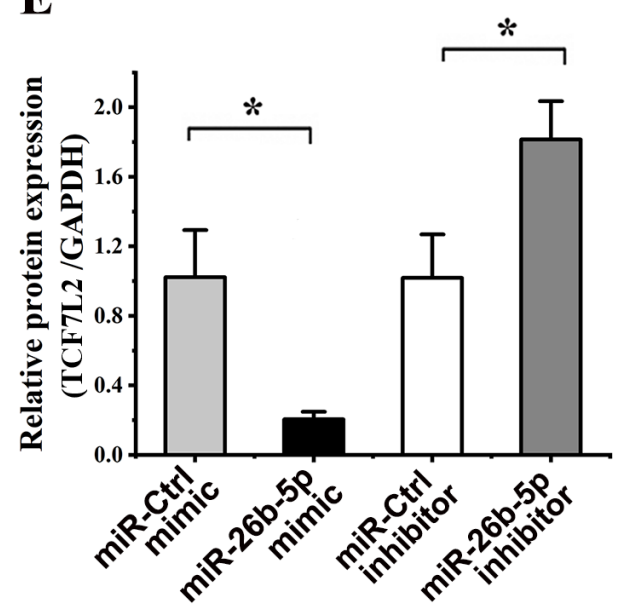

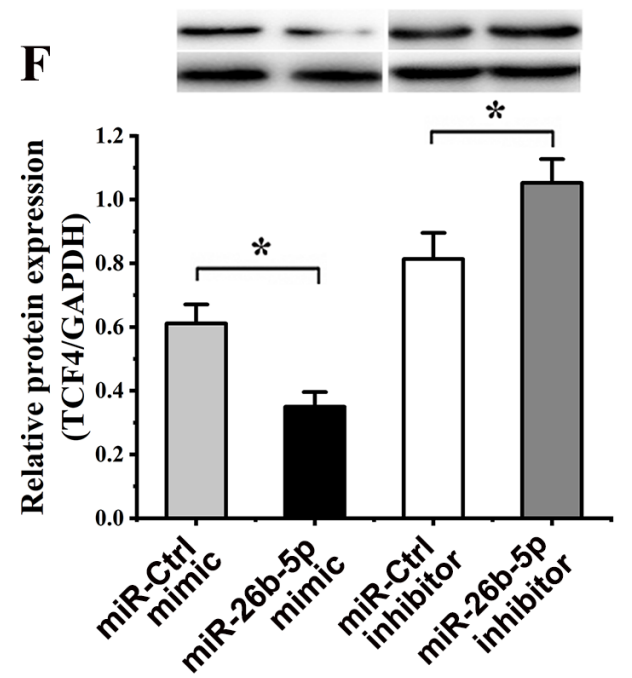

Figure 3

Binding between miR-26b-5p and TCF7L2. (A) The sequences of miR-26b-5p across species. (B) Binding between miR-26b-5p and 3'-UTR of TCF7L2. (C) Results of dual-luciferase reporter assays. The luciferase activity of hADMSCs transfected with miR-26b-5p mimic decreased $1.99 \pm 0.30$-fold $(p=0.002)$, comparing to miR-Ctrl inhibitor. The luciferase activity of hADMSCs transfected with miR-26b-5p inhibitor was not significantly change $(p=0.15)$, comparing to the reporter gene for TCF7L2 mRNA 3'-UTR 
mutated. (D) Results of dual-luciferase reporter assays. The luciferase activity of hADMSCs transfected with miR-26b-5p inhibitor increased $1.36 \pm 0.83$-fold $(p=0.036)$, comparing to the control group. The luciferase activity of hADMSCs transfected with miR-26b-5p inhibitor was not significantly change, comparing to the reporter gene for TCF7L2 mRNA 3'-UTR mutated. (E) qPCR analysis for TCF7L2 mRNA expression in hADMSCs transfected with miR-26b-5p mimic and inhibitor. TCF7L2 were decreased $5.00 \pm$ 0.64 -fold $(p=0.007)$ in hADMSCs transfected with miR-26b-5p mimic, comparing to hADMSCs transfected with miR-Ctrl mimic. TCF7L2 were increased $1.78 \pm 0.57$-fold $(p=0.014)$ in hADMSCs transfected with miR-26b-5p inhibitor, comparing to hADMSCs transfected with miR-Ctrl inhibitor. (F) Western blot analysis for TCF-4 protein expression in hADMSCs transfected with miR-26b-5p mimic and inhibitor. TCF-4 was decreased $1.75 \pm 0.10$-fold $(p=0.004)$ in hADMSCs transfected with miR-26b-5p mimic, comparing to hADMSCs transfected with miR-Ctrl mimic. TCF-4 was increased $1.29 \pm 0.08$-fold ( $p$ $=0.020)$ in hADMSCs transfected with miR-26b-5p inhibitor, comparing to hADMSCs transfected with miR-Ctrl inhibitor. 

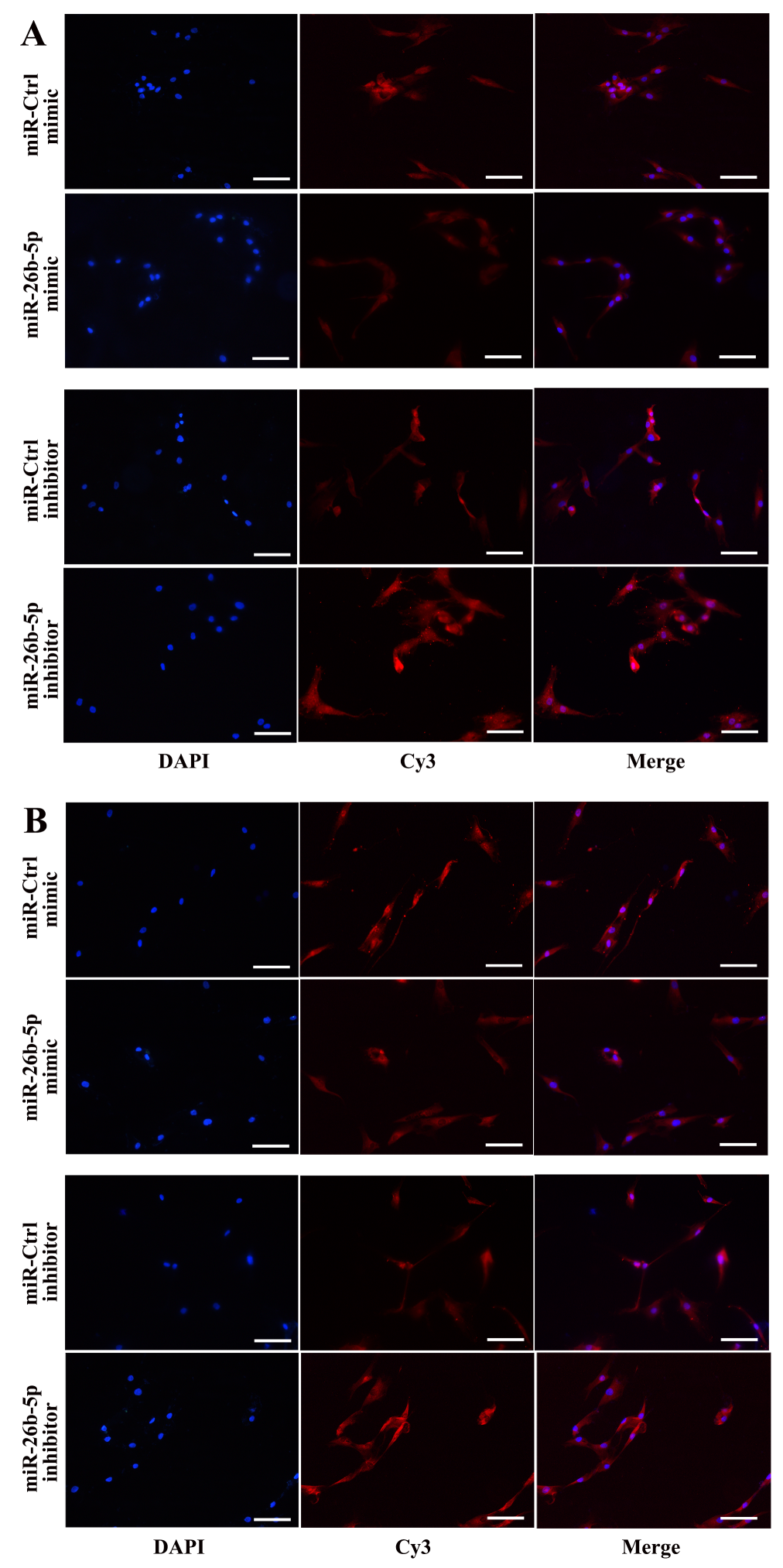

\section{Figure 4}

The immunofluorescence of TCF- 4 and $\beta$-catenin regulated by miR-26b-5p mimics and inhibitor. (A) The immunofluorescence intensity of TCF 4 and $\beta$-catenin were inhibited by miR-26b-5p mimic than the normal hADMSCs. (B) The immunofluorescence intensity of TCF4 and $\beta$-catenin were enhanced by miR-

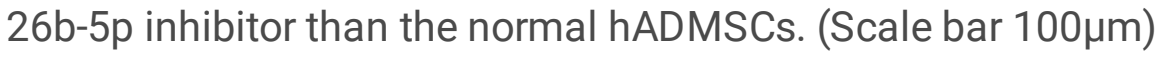



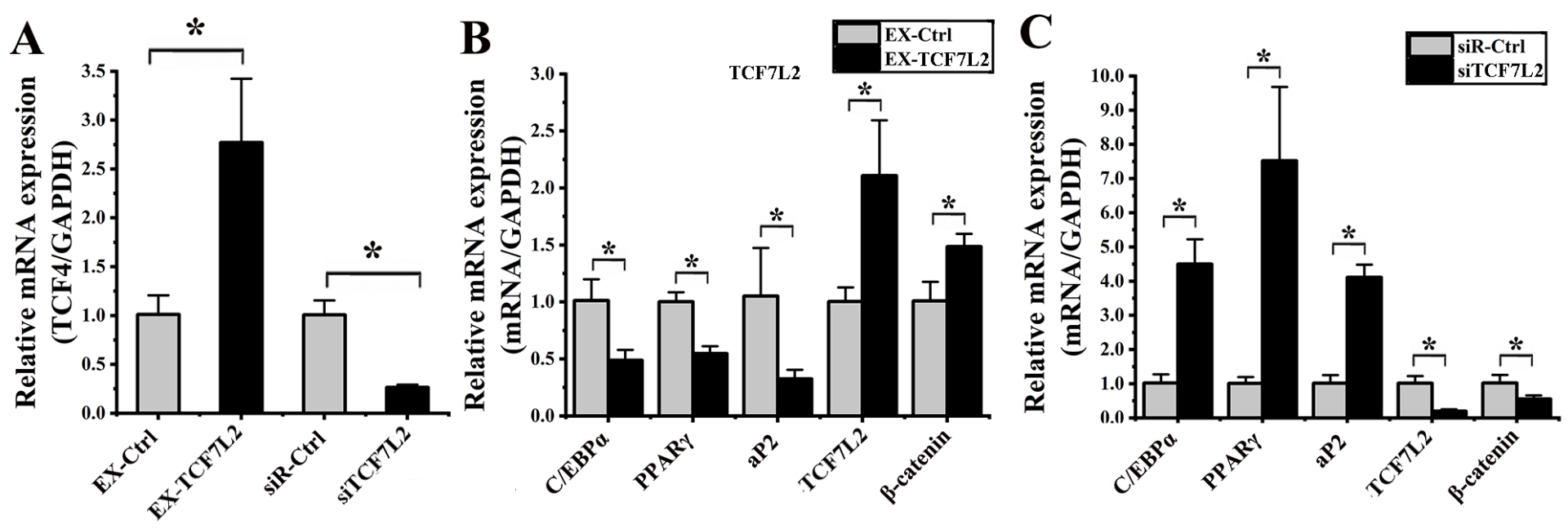

Figure 5

TCF-4 affects adipogenic differentiation of hADMSCs. ( ${ }^{*} p<0.05$, there were significant difference between these two groups) (A) Transfection efficiency. TCF7L2 mRNA expression in EX-TCF7L2 group increased $2.74 \pm 1.09$-fold $(p=0.011)$, comparing to the EX-Ctrl group. TCF7L2 mRNA expression in siTCF7L2 group decreased $3.81 \pm 0.54$-fold $(p=0.001)$, compared to the siR-Ctrl group. (B) Results of qPCR for overexpression of TCF7L2. Comparing to the EX-Ctrl group, the following mRNAs in transfecting EX-TCF7L2 in hADMSCs were decreased significantly: C/EBP a $2.07 \pm 0.35$-fold ( $p=0.012)$, PPARy $1.83 \pm$ 0.38 -fold $(p=0.002)$, aP2 $3.23 \pm 1.38$-fold $(p=0.043)$. And the following mRNAs were increased significantly: TCF7L2 $2.10 \pm 0.68$-fold $(p=0.019), \beta$-catenin $1.47 \pm 0.22$-fold $(p=0.015)$. (C) Results of qPCR for suppression of TCF7L2. Comparing to the siR-Ctrl group, the following mRNAs in transfecting siTCF7L2 in hADMSCs were increased significantly: C/EBP a $4.40 \pm 0.66$-fold $(p=0.043)$, PPARY $7.03 \pm$ 3.25 -fold $(p=0.034)$, aP2 $4.04 \pm 0.62$-fold $(p<0.008)$. And the following mRNAs were decreased significantly: TCF7L2 $5.11 \pm 1.52$-fold $(p=0.003)$, $\beta$-catenin $1.85 \pm 0.24$-fold $(p=0.037)$.
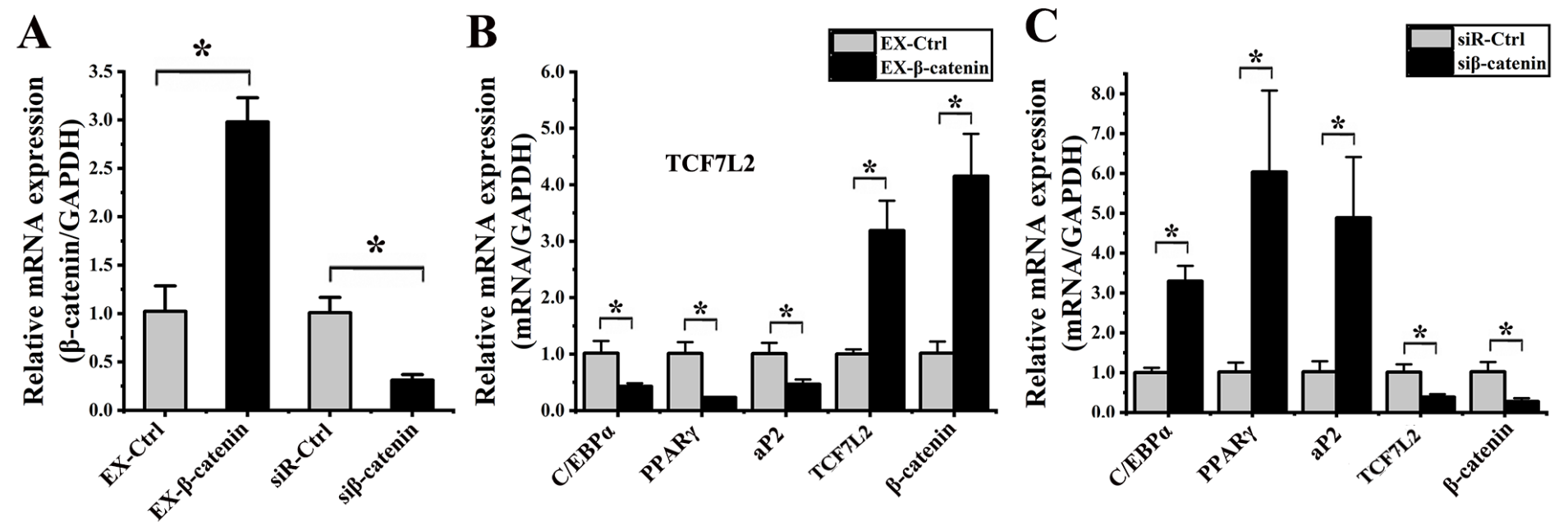

Figure 6 
$\beta$-catenin affects adipogenic differentiation of hADMSCs. $\left({ }^{*} p<0.05\right.$, there were significant difference between these two groups) (A) Transfection efficiency. $\beta$-catenin mRNA expression in EX- $\beta$-catenin group increased $2.92 \pm 0.84$-fold $(p=0.001)$, comparing to the EX-Ctrl group. $\beta$-catenin mRNA expression in si $\beta$ catenin group decreased $3.22 \pm 0.55$-fold $(p=0.002)$, compared to the siR-Ctrl group. (B) Results of $q P C R$ for overexpression of $\beta$-catenin. Comparing to the EX-Ctrl group, the following mRNAs in transfecting EXTCF7L2 in hADMSCs were decreased significantly: C/EBP a $2.36 \pm 0.78$-fold $(p=0.010)$, PPARY $4.32 \pm$ 0.98 -fold $(p=0.003)$, aP2 $2.16 \pm 0.87$-fold $(p=0.010)$. And the following mRNAs were increased significantly: TCF7L2 $3.18 \pm 0.69$-fold $(p=0.002), \beta$-catenin $4.09 \pm 0.64$-fold $(p=0.002)$. (C) Results of qPCR for suppression of $\beta$-catenin. Comparing to the siR-Ctrl group, the following mRNAs in transfecting si $\beta$-catenin in hADMSCs were increased significantly: C/EBP a $3.28 \pm 0.33$-fold $(p=0.001)$, PPARY $5.93 \pm$ 0.96 -fold $(p=0.013)$, aP2 $4.78 \pm 1.54$-fold $(p=0.012)$. And the following mRNAs were decreased significantly: TCF7L2 $2.61 \pm 0.54$-fold $(p=0.007), \beta$-catenin $3.67 \pm 1.73$-fold $(p=0.008)$.

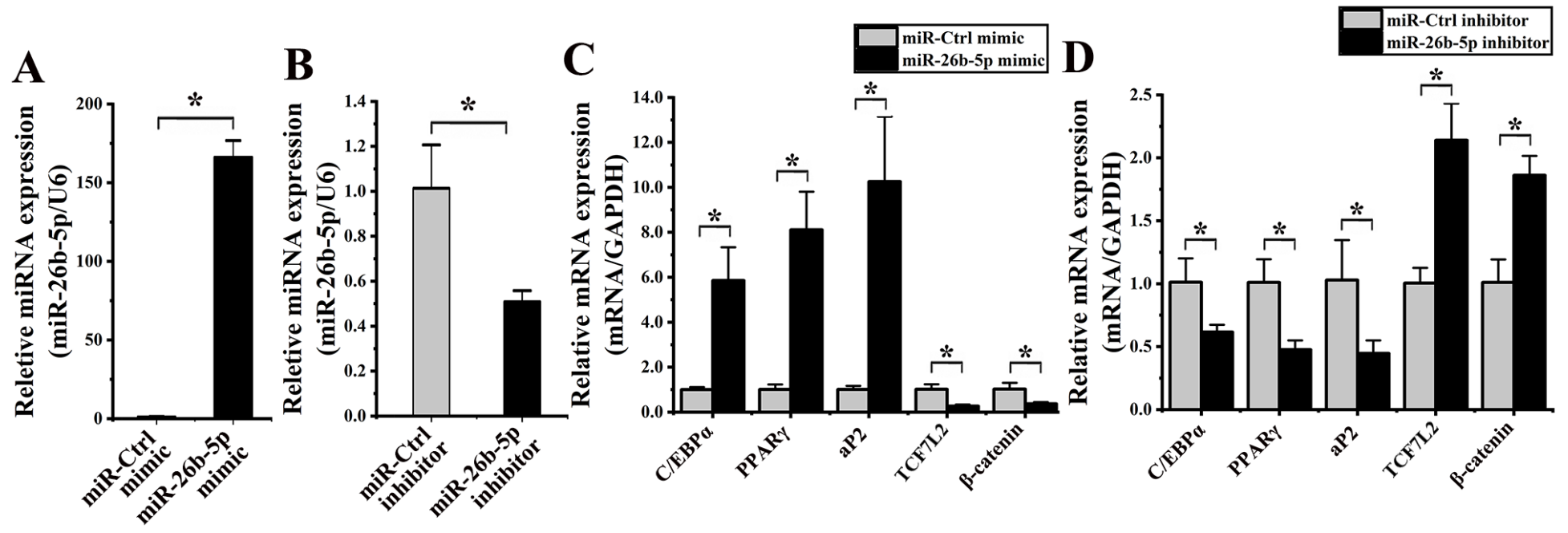

\section{Figure 7}

miR-26b-5p affects adipogenic differentiation of hADMSCs. ( ${ }^{\star} p<0.05$, there were significant difference between these two groups) (A) Transfection efficiency. miR-26b-5p expression in miR-26b-5p mimic group increased $161.15 \pm 41.26$-fold ( $<<0.001)$, comparing to the miR-Ctrl mimic group. miR-26b-5p expression in miR-26b-5p inhibitor group decreased $3.88 \pm 0.20$-fold $(p=0.012)$, compared to the miR-Ctrl inhibitor group. (B) Results of qPCR for overexpression of miR-26b-5p. Comparing to the miR-Ctrl mimic group, the following mRNAs in transfecting miR-26b-5p mimic in hADMSCs were increased significantly: C/EBP a $5.83 \pm 1.99$-fold $(p=0.029)$, PPARy $3.99 \pm 3.14$-fold $(p=0.002)$, aP2 $10.17 \pm 4.81$-fold $(p=0.005)$. And the following mRNAs were decreased significantly: TCF7L2 $3.80 \pm 1.96$-fold $(p=0.006), \beta$-catenin $2.80 \pm$ 1.34-fold $(p=0.016)$. (C) Results of qPCR for suppression of miR-26b-5p. Comparing to the miR-Ctrl inhibitor group, the following mRNAs in transfecting miR-26b-5p inhibitor in hADMSCs were decreased significantly: C/EBP a $1.64 \pm 0.47$-fold $(p=0.026)$, PPARY $2.12 \pm 0.71$-fold $(p=0.009)$, aP2 $2.31 \pm 0.72$ fold $(p=0.039)$. And the following mRNAs were increased significantly: TCF7L2 $2.13 \pm 0.36$-fold $(p=$ $0.003)$, $\beta$-catenin $1.84 \pm 0.28$-fold $(p=0.003)$. 

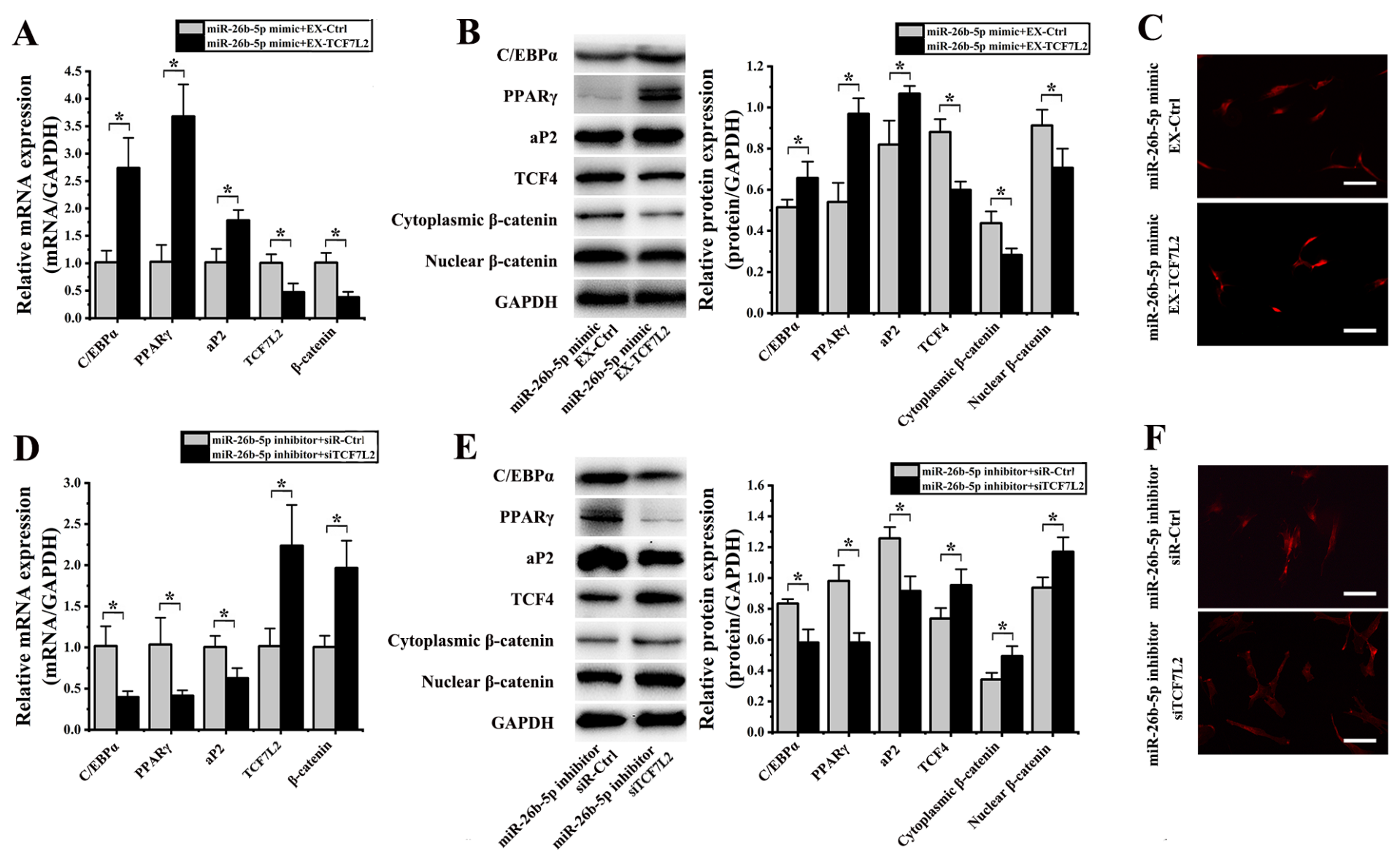

Figure 8

TCF4 regulated by miR-26b-5p influencing the adipogenic differentiation of hADMSCs. (A) Results of qPCR for overexpression of miR-26b-5p and TCF7L2. Comparing to hADMSCs cotransfected with miR26b-5p mimic and EX-Ctrl group, the following mRNAs in transfecting miR-26b-5p mimic and EX-TCF7L2 in hADMSCs were increased significantly: C/EBP a $2.69 \pm 0.10$-fold $(p=0.007)$, PPARy $3.58 \pm 1.47$-fold ( $p$ $=0.002)$, aP2 $1.75 \pm 0.55$-fold $(p=0.013)$. And the following mRNAs were decreased significantly: TCF7L2 $2.13 \pm 0.99$-fold ( $p=0.014)$, $\beta$-catenin $2.65 \pm 0.31$-fold $(p=0.006)$. (B) Western blot analysis for protein expression in hADMSCs overexpressed miR-26b-5p and TCF7L2. Comparing to hADMSCs cotransfected with miR-26b-5p mimic and EX-Ctrl group, the following proteins in hADMSCs cotransfected with miR-26b-5p mimic and EX-TCF7L2 were increased significantly: C/EBP a $1.28 \pm 0.08$ fold $(p=0.048)$, PPARy $1.79 \pm 0.21$-fold $(p=0.004)$, aP2 $1.30 \pm 0.19$-fold $(p=0.025)$. And the following proteins were decreased significantly: TCF-4 $1.47 \pm 0.09$-fold $(p=0.003)$, cytoplasmic $\beta$-catenin $1.55 \pm$ 0.03-fold ( $p=0.015$ ), nuclear $\beta$-catenin $1.29 \pm 0.07$-fold ( $p=0.042)$. (C) After transfected with miR-26b-5p mimic and EX-TCF7L2, the fluorescence intensity decreased in the nucleus. (D) Results of qPCR for suppression of miR-26b-5p and TCF7L2. Comparing to hADMSCs cotransfected with miR-26b-5p inhibitor and siR-Ctrl group, the following mRNAs in hADMSCs cotransfected with miR-26b-5p inhibitor and siTCF7L2 were increased significantly: C/EBP a $2.56 \pm 1.23$-fold $(p=0.013)$, PPARY $2.51 \pm 0.94$-fold $(p=0.031)$, aP2 $1.60 \pm 0.54$-fold $(p=0.023)$. And the following mRNAs were increased significantly: TCF7L2 $2.20 \pm 0.09$-fold $(p=0.017), \beta$-catenin $1.95 \pm 0.46$-fold $(p=0.010)$. (E) Western blot analysis for 
protein expression in hADMSCs suppressed miR-26b-5p and TCF7L2. Comparing to hADMSCs cotransfected with miR-26b-5p inhibitor and siR-Ctrl group, the following proteins in hADMSCs cotransfected with miR-26b-5p inhibitor and siTCF7L2 were decreased significantly: C/EBP a 1.43 \pm 0.21fold ( $p=0.008)$, PPARy $1.68 \pm 0.05$-fold ( $p=0.004)$, aP2 $1.37 \pm 0.13$-fold $(p=0.008)$. And the following proteins were increased significantly: TCF-4 $1.29 \pm 0.14$-fold $(p=0.039)$, cytoplasmic $\beta$-catenin $1.44 \pm$ 0.20 -fold ( $p=0.028)$, nuclear $\beta$-catenin $1.25 \pm 0.16$-fold $(p=0.025)$. ( $F$ ) After transfected with miR-26b-5p inhibitor and siTCF7L2, the fluorescence intensity decreased in the nucleus.
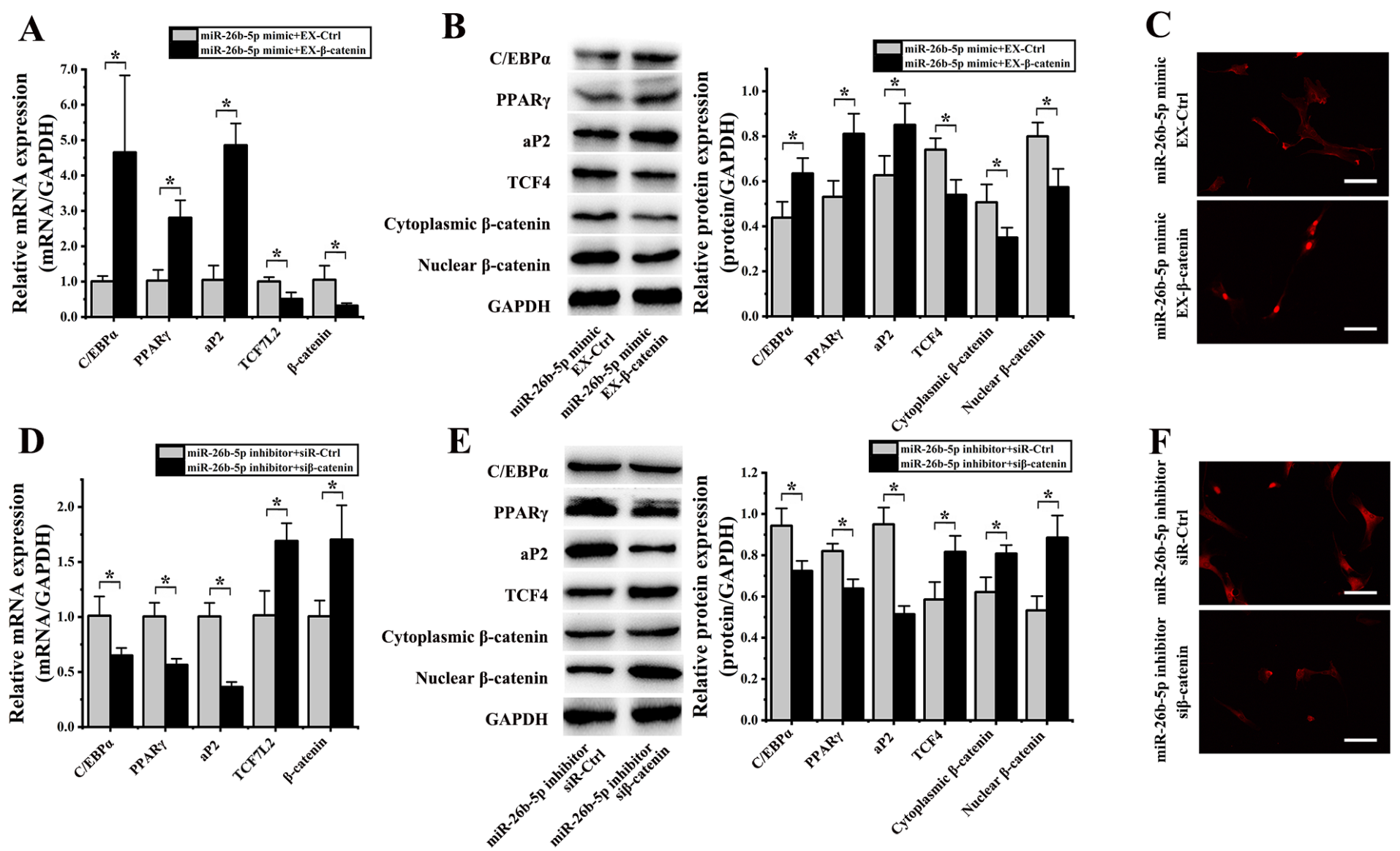

\section{Figure 9}

$\beta$-catenin regulated by miR-26b-5p influencing the adipogenic differentiation of hADMSCs. (A)Results of qPCR for overexpression of miR-26b-5p and $\beta$-catenin. Comparing to hADMSCs cotransfected with miR26b-5p mimic and EX-Ctrl group, the following mRNAs in transfecting miR-26b-5p mimic and EX- $\beta$-catenin in hADMSCs were increased significantly: C/EBP a $4.62 \pm 1.78$-fold ( $p=0.004$ ), PPARy $2.73 \pm 0.37$-fold ( $p$ $=0.007)$, aP2 $4.64 \pm 2.16$-fold $(p=0.001)$. And the following mRNAs were decreased significantly: TCF7L2 $1.97 \pm 0.82$-fold ( $p=0.019$ ), $\beta$-catenin $3.30 \pm 0.52$-fold ( $p=0.036)$. (B) Western blot analysis for protein expression in hADMSCs overexpressed miR-26b-5p and $\beta$-catenin. Comparing to hADMSCs cotransfected with miR-26b-5p mimic and EX-Ctrl group, the following proteins in hADMSCs cotransfected with miR-26b-5p mimic and EX- $\beta$-catenin were increased significantly: C/EBP a 1.45 \pm 0.07fold ( $p=0.027$ ), PPARy $1.53 \pm 0.10$-fold ( $p=0.013)$, aP2 $1.36 \pm 0.14$-fold $(p=0.040)$. And the following proteins were decreased significantly: TCF-4 1.37 \pm 0.19 -fold $(p=0.014)$, cytoplasmic $\beta$-catenin $1.45 \pm$ 
0.16-fold $(p=0.041)$, nuclear $\beta$-catenin $1.39 \pm 0.12$-fold $(p=0.018)$. (C) After transfected with miR-26b-5p mimic and EX- $\beta$-catenin, the fluorescence intensity decreased in the nucleus. (D) Results of qPCR for suppression of miR-26b-5p and $\beta$-catenin. Comparing to hADMSCs cotransfected with miR-26b-5p inhibitor and siR-Ctrl group, the following mRNAs in hADMSCs cotransfected with miR-26b-5p inhibitor and si $\beta$-catenin were increased significantly: C/EBP a $1.56 \pm 0.17$-fold $(p=0.030)$, PPARy $1.78 \pm 0.37$-fold $(p=0.005)$, aP2 $2.76 \pm 0.69$-fold $(p=0.001)$. And the following mRNAs were increased significantly: TCF7L2 $1.67 \pm 0.31$-fold $(p=0.013)$, $\beta$-catenin $1.69 \pm 0.51$-fold $(p=0.024)$. (E) Western blot analysis for protein expression in hADMSCs suppressed miR-26b-5p and $\beta$-catenin. Comparing to hADMSCs cotransfected with miR-26b-5p inhibitor and siR-Ctrl group, the following proteins in hADMSCs cotransfected with miR-26b-5p inhibitor and si $\beta$-catenin were decreased significantly: C/EBP a $1.30 \pm$ 0.05 -fold $(p=0.005)$, PPARy $1.29 \pm 0.03$-fold $(p=0.017)$, aP2 $1.85 \pm 0.02$-fold $(p=0.001)$. And the following proteins were increased significantly: TCF-4 $1.39 \pm 0.07$-fold $(p=0.026)$, cytoplasmic $\beta$-catenin $1.30 \pm 0.08$-fold $(p=0.018)$, nuclear $\beta$-catenin $1.66 \pm 0.09$-fold $(p=0.009)$. ( $F)$ After transfected with miR$26 b-5 p$ inhibitor and si $\beta$-catenin, the fluorescence intensity decreased in the nucleus. 